Powell, E. O. (1956). J. gen. Microbiol. 14, 153-159

\title{
A Rapid Method for Determining the Proportion of Viable Bacteria in a Gulture
}

\author{
By E. O. POWELL \\ Microbiological Research Department, Ministry of Supply, \\ Porton, Wiltshire
}

SUMMARY: A method of making graticules on the surface of Cellophane is described. The proportion of viable bacteria in a culture can be estimated by inoculating a sample on to such a graticule, and counting the organisms before and after a short period of growth.

The usual plating-out method of obtaining a viable count of bacteria is expensive of both time and materials, and the result is obtained only after a period of incubation never less than $12 \mathrm{hr}$. The result could obviously be established more quickly by counting the organisms in an inoculum under the microscope, and again counting those which had multiplied after a relatively short period of growth. The practical difficulty in doing so is that only a few organisms can comfortably be accommodated in a single field of the microscope, if confusion of one micro-colony with another is to be avoided. Some form of counting-chamber is required, to enable a definite, recognizable, and sufficiently large area to be scanned before and after the growth period. It is now well known that most common bacteria will grow freely on the surface of a Cellophane membrane through which they draw their nourishment, and Pearce \& Powell (1951) and Harris \& Powell (1951) have shown also that Cellophane is particularly suitable as a support for growing organisms under the microscope. A rapid method for estimating a viable count, described in the following paragraphs, consists in growing the organisms on a Cellophane membrane lying on the surface of a suitable agar medium. Its practicability depends on a simple device for engraving a graticule on the membrane, so as to meet the difficulty mentioned above. The development of this method was occasioned during the study of continuous culture of the Monod (1950) type. Most of the variables of interest in such a study (e.g. pH, temperature, flow rate) can be determined almost instantaneously, and applied to automatic monitoring and regulation of the culture. This cannot yet be done with the viable count, but the early detection of changes in viability is still a great economy in the correction of unforeseen faults and in determining the effects of experimental variation in working conditions.

\section{Construction and use of the engraving tool}

Razor blades of about $100 \mu$. thickness are marketed by several manufacturers (Thin Gillette blades were used here). A pile of such blades, impressed twice on a Cellophane surface, one impression at right angles to the other, produces a 
grid of which one square is conveniently covered by a single field of a $4 \mathrm{~mm}$. microscope objective with a $\times 10$ eyepiece. The blade edges are not straight, but have a gentle undulation with an amplitude of $10-20 \mu$. in the plane of the blade; a tolerably uniform grid can only be produced if the blades are mounted so that the edges are in register, within a few microns, over the length of the graticule edge ( $1 \mathrm{~mm}$. is sufficient). The required accuracy of setting is achieved
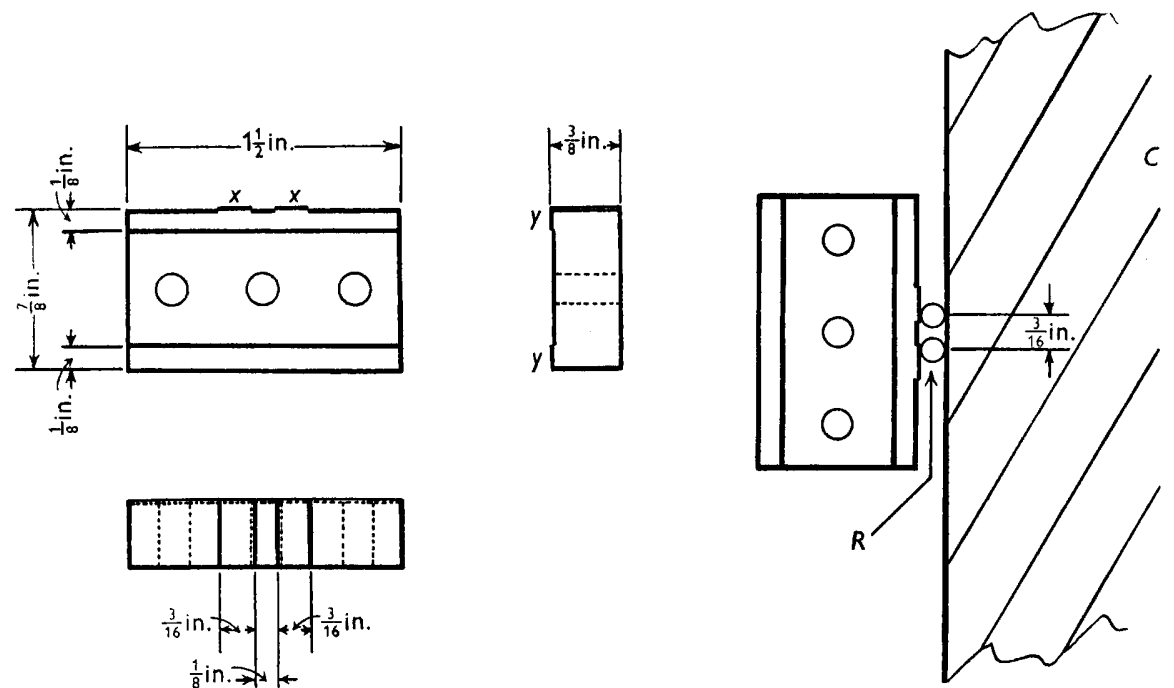

Fig. 1

Fig. 2

Fig. 1. Mild steel block. Two are used for clamping the pile of razor blades. The lands $x x$, $y y$ project about $0 \cdot 005$ in. above the neighbouring surface.

Fig. 2. Preparation for mounting blades. $C$, body of magnetic chuck; $R$, rollers. Viewed from above.

by clamping the blades between two mild steel blocks of the form shown in Fig. 1. These blocks are of approximately the same size (in side elevation) as the blades; on two surfaces of each, lands $x x$, yy are left about 0.005 in. proud of the neighbouring surface. After being machined, the two blocks are bolted together and the lands $x x$ finish-ground so that all four are in the same plane.

The blades are positioned as follows: two silver steel rods $1 \frac{1}{2}$ in. long and $\frac{1}{8}$ in. diameter, ground and polished, are clamped to the surface of a magnetic chuck with their axes vertical, approximately parallel and $\frac{3}{16}$ in. apart. They are prevented from rolling by an application of plasticine to their ends. One of the blocks is then placed with its lands $x x$ in contact with the lower ends of the rollers and the lands yy upwards (Fig. 2); the field of the chuck is sufficient to hold it in position. The blades, conveniently eleven in number, are placed one by one on the block; again the field keeps their edges in contact with the rollers. Some delicacy of touch is needed to prevent the edges being turned by longitudinal rubbing. The second block is then similarly brought into contact with the upper ends of the rollers, and slid downwards upon the stack 
of blades. The whole pile is bolted firmly together, and the blade edges between the lands are then in the same plane as the lands themselves.

The second member of the tool, the anvil, which determines the depth of the impression, consists of a glass plate painted with label varnish which is scraped away except for a transverse strip $\frac{3}{32}$ in. wide. The correct thickness, 10-12 $\mu$. must be reached by trial, the varnish being diluted when necessary. It can be estimated quite accurately enough on an ordinary micrometer. The plate is hinged by means of adhesive tape to one edge of the working face of the pile so that the strip of varnish lies opposite the gap between the lands $x x($ Pl. 1 $a)$.

The pile must be washed in light petroleum before use, in order to remove grease and dust.

A graticule is made by placing a disk of Cellophane centrally on the pile of blades, bringing down the glass plate over it, and pressing fairly heavily with the thumb; this is repeated after turning the disk through a right angle. The disk is marked at some point on its edge so that it can be brought into the same azimuth whenever it is examined under the microscope. Under a low power (Pl. 1c) the graticule looks unpromisingly dirty; this is due to the small aperture of the objective, which increases contrast and, because of the large focal depth, shows up every inequality within the thickness of the membrane and part of the underlying agar. At a higher power ( $\mathrm{Pl} .1 d)$, the surface is seen to be mostly free from gross irregularity. Such a graticule can be made in about $1 \mathrm{~min}$., inclusive of punching out the disk, and so it can economically be discarded after use. The useful life of a pile of blades is not known-many hundred impressions at least can be made without loss of edge.

Ordinary commercial Cellophane is suitable for this application (no. PT. 300, Messrs British Cellophane Ltd., Bridgwater, Somerset). As it comes from the manufacturer it is physically clean and sterile over large areas; for the present purpose freedom from grease and dust is much more important than asepsis, so the Cellophane should be kept wrapped and handled only with forceps. When slow-growing organisms are to be examined, contamination can in nearly every instance be avoided by washing the completed graticule in boiling water and laying it while still wet on the agar plate.

\section{Method for determining the proportion of viable organisms in an inoculum}

To make a count, a graticule with the engraved side uppermost is placed on the surface of a nutrient agar plate. The centre of the disk is inoculated by means of a $1 \mathrm{~mm}$. loop with a suspension of organisms diluted to $10^{6}-10^{7} / \mathrm{ml}$. The inoculated area is examined under vertical illumination with a dry $4 \mathrm{~mm}$. metallurgical objective. To hasten drying and permit the organisms to be seen clearly, a stream of air is blown over the surface; $\mathrm{Pl} .16$ shows a jet clipped to the objective mount for this purpose. When all the liquid is evaporated, the current of air is decreased as far as possible without allowing the liquid diffusing through the Cellophane to flood the individual organisms (Pl. 1d; cf. Harris \& Powell, 1951). The graticule is scanned square by square; the number 
of single organisms and of groups in each is noted down. Squares in which the count is embarrassed by dirt, objects of doubtful character, overcrowding, or blemishes in the 'Cellophane', can be omitted. After incubation for 2-6 hr. (depending on the organism) the numbers of groups of organisms in each square are again counted. The difference in the group count is the number of viable organisms among the single individuals originally present. This method of counting is adopted because non-viable cells sometimes undergo lysis and leave little residue. If single organisms only were counted, such cells would be recorded as viable. The proportion of viable organisms so obtained must of course be supplemented by a conventional total count if a measure of their actual concentration is required.

Much the best results are obtained if these operations are performed throughout at the temperature of incubation. The dilution and inoculation of the sample at that temperature keeps the lag period to a minimum and the more rapid drying of the inoculum reduces the risk of damage to the organisms. There is a danger that liquid may diffuse to the surface of the 'Cellophane' in sufficient quantity to permit the organisms to move during incubation. This can be avoided by drying the agar plates for $2 \mathrm{hr}$. at $37^{\circ}$ beforehand, and incubating with the lids slightly ajar.

In practice it is found to be nearly always quite easy to distinguish organisms from foreign bodies, though some previous experience is of course desirable. It is impossible to convey in a photomicrograph the nuances obtainable by minute adjustments of focus and of degree of dryness. It is rare to find pairs of organisms in close juxtaposition but not touching. As the inoculum dries, organisms which are only a few diameters apart are drawn together by surface tension. Unless the inoculum is very dense (e.g. $>10$ organisms/square), the error which can arise by fusion of adjacent colonies is thus small.

\section{Appraisal of the method}

The method has been applied so far to a limited range of organisms only: Escherichia coli, Pasteurella pestis, Serratia marcescens, Pseudomonas aeruginosa, several Bacillus species; but organisms of many other genera are known to grow satisfactorily on Cellophane. The numerical results are reproducible and agree (except in the special cases discussed below) with those obtained by the normal procedure when due allowance for sampling fluctuations is made (Table 1). There are four principal advantages, apart from speed: (i) Directness - the organisms capable of growing in the circumstances of the test are actually seen to do so; viability is not asserted as an induction from some concomitant property. (ii) A test sample can be transferred within a minute to the graticule. At most one dilution (which can usually be guessed) is needed to bring the organisms to a density convenient for counting. If a culture containing many ill-nourished or unhealthy organisms is subjected to a lengthy process of dilution, some will die meanwhile, whereas rapid transfer to fresh medium leaves less opportunity for irreparable damage to occur. (iii) Errors due to clumping of the organisms are avoided. (iv) Those organisms (especially 
certain Bacillus spp.) which form diffuse or spreading colonies and cannot be satisfactorily counted by the ordinary method, offer no special difficulty (Table 1).

Table 1. Proportion of viable organisms in various cultures, estimated by counting on 'Cellophane' graticules

\begin{tabular}{|c|c|c|c|c|c|}
\hline $\begin{array}{l}\text { Nature } \\
\text { of } \\
\text { inoculum }\end{array}$ & $\begin{array}{l}\text { Period of } \\
\text { incubation } \\
\quad(\mathrm{hr} .)\end{array}$ & $\begin{array}{l}\text { No. of single } \\
\text { organisms } \\
\text { counted }\end{array}$ & $\begin{array}{c}\text { No. of } \\
\text { viable } \\
\text { organisms }\end{array}$ & $\begin{array}{c}\% \\
\text { viable }\end{array}$ & $\begin{array}{c}\% \text { viable } \\
\text { by } \\
\text { plating-out }\end{array}$ \\
\hline $\begin{array}{l}\text { Artificial mixture of } \\
50 \% \text { viable, } 50 \% \text { heat- } \\
\text { killed } \text { Escherichia coli }\end{array}$ & ti & 729 & 376 & 52 & $(50)$ \\
\hline $\begin{array}{l}\text { Bacillus anthracis } \\
\text { spores. }\end{array}$ & $2 \frac{1}{2}$ & $\begin{array}{r}86 \\
245 \\
122 \\
65 \\
102 \\
76\end{array}$ & $\begin{array}{r}79 \\
229 \\
109 \\
65 \\
96 \\
65\end{array}$ & $\begin{array}{r}92 \\
94 \\
89 \\
100 \\
94 \\
86\end{array}$ & 一 \\
\hline $\begin{array}{l}\text { Total of previous six } \\
\text { counts }\end{array}$ & - & 696 & 643 & 92 & 90 \\
\hline Pasteurella pestis. & 5 & $\begin{array}{l}154 \\
128 \\
124 \\
141 \\
137 \\
150\end{array}$ & $\begin{array}{l}131 \\
101 \\
114 \\
127 \\
112 \\
133\end{array}$ & $\begin{array}{l}85 \\
79 \\
92 \\
90 \\
82 \\
88\end{array}$ & - \\
\hline $\begin{array}{l}\text { Total of previous six } \\
\text { counts }\end{array}$ & 一 & 834 & 718 & 86 & 90 \\
\hline $\begin{array}{l}\text { Bacillus cereus spores. } \\
\text { A strain giving } \\
\text { spreading colonies. }\end{array}$ & 3 & $\begin{array}{l}213 \\
239 \\
219 \\
108\end{array}$ & $\begin{array}{l}209 \\
222 \\
214 \\
101\end{array}$ & $\begin{array}{l}98 \\
93 \\
98 \\
94\end{array}$ & $\begin{array}{c}\text { c. } 50 \\
\text { (impossible } \\
\text { to count } \\
\text { accurately) }\end{array}$ \\
\hline $\begin{array}{l}\text { Bacillus globigii spores } \\
\text { incorporating radio } \\
\text { phosphorus. }\end{array}$ & 3 & $\begin{array}{l}473 \\
309\end{array}$ & $\begin{array}{l}30 \\
22\end{array}$ & $\begin{array}{l}6 \cdot 3 \\
7 \cdot 1\end{array}$ & $7 \cdot 0$ \\
\hline $\begin{array}{l}\text { Aerobacter aerogenes } \\
\text { samples from con- } \\
\text { tinuous culture. }\end{array}$ & 2 & $\begin{array}{l}121 \\
125 \\
151\end{array}$ & $\begin{array}{l}106 \\
109 \\
132\end{array}$ & $\begin{array}{l}88 \\
87 \\
87\end{array}$ & 74 \\
\hline
\end{tabular}

Notes (1) For the purposes of this trial, many of the above suspensions were diluted more than is normally necessary.

(2) Pasteurella pestis was grown on a tryptic meat medium with an addition of $0.075 \%$ sodium bisulphite. The individual counts are discordant (in the statistical sense) at the $5 \%$ level of significance. A similar lack of reproducibility occurs in the conventional method of counting, and is no doubt connected with the difficulty of establishing growth from single isolated cells (Herbert, 1949).

(3) A differential staining method (Powell, 1950) showed that about $95 \%$ of the Bacillus cereus spores tested were ungerminated, and presumably viable.

(4) For preparation and properties of spores containing ${ }^{32} \mathrm{P}$ see Harper \& Morton (1952).

The main disadvantages are: (i) The limited statistical accuracy. Only a few hundred organisms can conveniently be counted on one graticule. However, it is often possible to inoculate several graticules on one agar plate at the same time. (ii) Some organisms, ultimately capable of reproduction, may have a very long lag period on transfer to a new medium, and so may be accounted 
non-viable. Increasing the period of incubation is of no avail if the dispersion of lag is large, because the colonies formed from the organisms first to grow soon extend over a wide area. (iii) Some familiarity with the appearance, under vertical illumination, of different sorts of organisms and of common foreign particles is necessary if the observer is to have confidence in his assessment.

There have been proposed or implied several rapid methods of determining viability based on properties known to be highly correlated with the power of reproduction, e.g. differential staining (Strugger, 1948; Wade \& Morgan 1954); refractive index changes, visible under phase-contrast (Barer, Ross \& Tzaczyk, 1953); growth without division (Valentine \& Bradfield, 1953, 1954). Such methods work well with artificial mixtures of unequivocally living and dead organisms, but in natural mixtures there are often many intermediate forms which cannot be categorized with certainty in this way. This is the chief justification for introducing a method which requires specialized apparatus and which, though expeditious, must be painstakingly carried out if it is to yield reliable results.

It seemed at first that counting on the Cellophane surface would be facilitated by applying Valentine \& Bradfield's principle, i.e. by incorporating urea in the medium, so that division would be suppressed and viable organisms easily recognizable as long filaments. Trials with several species of organisms gave uniformly low results, evidently because evaporation at the surface raises the urea concentration to a toxic level there (cf. Harris \& Powell, 1951).

The Cellophane graticule technique has been found to give higher figures than the usual plating out in three sets of circumstances. (Compare also the discussion of Valentine \& Bradfield, 1954.) (i) In samples taken from continuous cultures of the Monod type (Table 1). The discrepancy is probably to be ascribed to the death of some organisms during the preparation of serial dilutions. In such cultures the growth-rate is controlled by limiting the supply of nutrient, i.e. by starving the organisms, so that they can be expected to have but little reserve of energy. The viability as estimated by the differential stain of Wade \& Morgan (1954) is even higher-nearly $100 \%$. (ii) Organisms which have been damaged by radiation may reproduce for a few generations, but never give rise to macroscopic colonies (Buckland, Harper \& Morton, 1950). (iii) In non-homogeneous cultures two organisms of slightly different growth-rates will give rise to colonies of which one may have reached a nearly static condition before the other has become visible. After only a few generations of growth the difference will not be clearly evident. A preparation of highly virulent spores of Bacillus anthracis gave an apparent viability of only $0 \cdot 1 \%$ by the usual method. Closer examination of the plates showed that many microscopic colonies accompanied the few of normal size. In this instance it is likely that the much higher figure obtained by growth on Cellophane was still too low.

Anomalies of this kind arise not from any genuine inconsistency, but because it is practically impossible in the present state of knowledge to set up a satisfactory definition of viability; for this reason no two ways of deter- 
Journal of General Microbiology, Vol. 14, No. 1
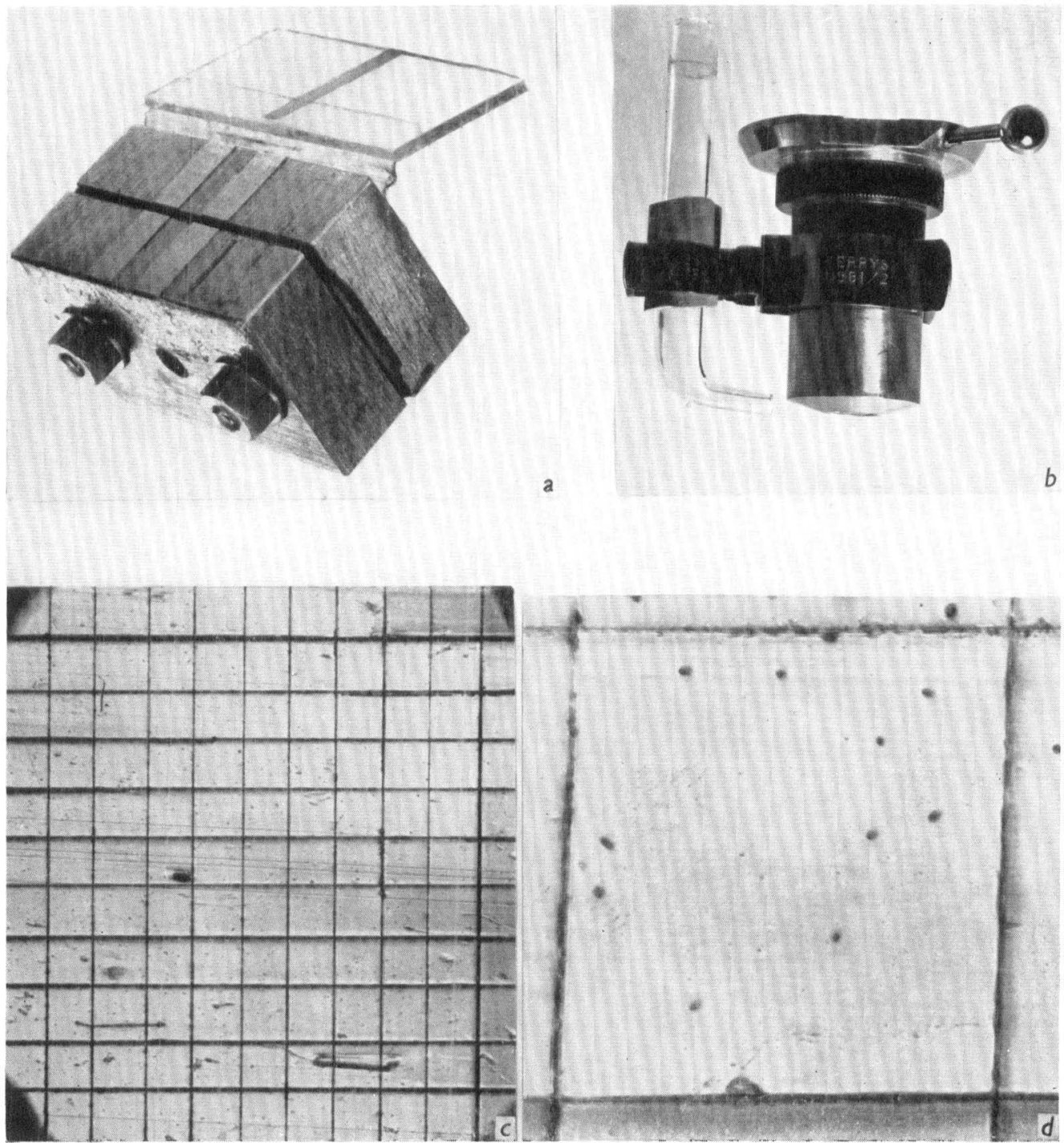

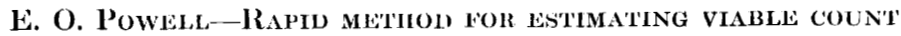


mining it will agree in every application. In principle, viability only has meaning with respect to an environment which can be specified. It is frequently unnecessary to take account of the restriction, but signal reminders occur from time to time, as in 'dormant' spores, or in Mycobacterium leprae (which has never been artificially cultivated). The work of Heinmets and collaborators (e.g. Heinmets, Taylor \& Lehman, 1953) on the resuscitation of 'dead' cells is a further reminder of the contingent nature of any possible definition.

I am indebted to my colleagues R. Elsworth, L. R. P. Meakin and R. C. Telling for information on samples taken from continuous cultures.

\section{REFERENCES}

Barer, R., Ross, K. A. F. \& Tzaczyk, S. (1953). Refractometry of living cells. Nature, Lond. 171, 720.

Buckland, F. E., Harper, G. J. \& Morton, J. D. (1950). Use of spores labelled with radiophosphorus in the study of respiratory retention of aerosols. Nature, Lond. 166, 354.

Harper, G. J. \& Morton, J. D. (1952). Bacillus subtilis spores labelled with radiophosphorus. J. gen. Microbiol. 7, 98.

Harris, N. K. \& Poweld, E. O. (1951). A culture chamber for the study of living bacteria, with some observations on the spore-bearing aerobes. J.R. micr. Soc. $71,407$.

Heinmets, F., Taylor, W. W. \& Lehman, J. J. (1953). The use of metabolites in the restoration of the viability of heat and chemically inactivated Escherichia coli. J. Bact. 67, 5.

Herbert, D. (1949). Studies on the nutrition of Pasteurella pestis and factors affecting the growth of isolated cells on an agar surface. Brit. J. exp. Path. 30, 509.

Monod, J. (1950). La technique de culture continue, théorie et applications. Ann. Inst. Pasteur, 79, 390.

Pearce, T. W. \& Powell, E. O. (1951). New techniques for the study of growing micro-organisms. J. gen. Microbiol. 5, 91.

Powell, J. F. (1950). Factors affecting the germination of thick suspensions of $B$. subtilis spores in L-alanine solution. J. gen. Microbiol. 4, 330.

StrugGer, S. (1948). Fluorescence microscope examination of bacteria in soil. Canad. J. Res. (G), 26, 188.

Valentine, R. C. \& Bradfield, J. R. G. (1953). A new procedure for bacterial viability counts and its biophysical application. Nature, Lond. 171, 878.

VAlentine, R. C. \& Bradfield, J. R. G. (1954). The urea method for bacterial viability counts with the electron microscope and its relation to other viability counting methods. J. gen. Microbiol. 11, 349.

Wade, H. E. \& Morgan, D. M. (1954). Differentiation of growing and non-growing bacteria by a staining technique. Nature, Lond. 174, 920.

\section{EXPLANATION OF PLATE}

(a) Complete engraving tool.

(b) Microscope objective with jet clipped on, for controlled drying.

(c) Low-power view of a graticule lying on an agar surface. The small squares are about $100 \mu$. across.

(d) High-power view of a single square of the graticule, with an inoculum of Serratia marcescens.

(Received 11 August 1955) 